\title{
A high-resolution VLT/FLAMES study of individual stars in the centre of the Fornax dwarf spheroidal galaxy ${ }^{\star}$ (Corrigendum)
}

\author{
B. Letarte ${ }^{1,2,3}$, V. Hill ${ }^{4,5}$, E. Tolstoy ${ }^{1}$, P. Jablonka ${ }^{4,6}$, M. Shetrone ${ }^{7}$, K. A. Venn ${ }^{8}$, M. Spite ${ }^{4}$, M. J. Irwin ${ }^{9}$, \\ G. Battaglia ${ }^{10}$, A. Helmi ${ }^{1}$, F. Primas ${ }^{10}$, P. François ${ }^{4}$, A. Kaufer ${ }^{11}$, T. Szeifert ${ }^{11}$, N. Arimoto ${ }^{12}$, and K. Sadakane ${ }^{13}$ \\ ${ }^{1}$ Kapteyn Astronomical Institute, University of Groningen, P.O. Box 800, 9700AV Groningen, The Netherlands \\ 2 California Institute of Technology, 1200 E. California Blvd, MC105-24, Pasadena, CA 91125, USA \\ ${ }^{3}$ South African Astronomical Observatory, P.O. Box 9, Observatory 7935, South Africa \\ e-mail: bruno@saao.ac.za \\ ${ }^{4}$ GEPI, Observatoire de Paris, CNRS, Université Paris Diderot, 92125 Meudon Cedex, France \\ ${ }^{5}$ Université de Nice Sophia-Antipolis, CNRS, Observatoire de Côte d'Azur, Laboratoire Cassiopée, 06304 Nice Cedex 4, France \\ ${ }^{6}$ Observatoire de Genève, Université de Genèva, 1290 Sauverny, Switzerland \\ ${ }^{7}$ McDonald Observatory, University of Texas, Fort Davis, TX 79734, USA \\ ${ }^{8}$ Dept. of Physics \& Astronomy, University of Victoria, 3800 Finerty Road, Victoria, BC V8P 1A1, Canada \\ ${ }^{9}$ Institute of Astronomy, University of Cambridge, Madingley Road, Cambridge CB3 OHA, UK \\ ${ }^{10}$ European Southern Observatory, Karl-Schwarzschild-str. 2, 85748 Garching bei München, Germany \\ ${ }^{11}$ European Southern Observatory, Alonso de Cordova, 3107 Santiago, Chile \\ ${ }^{12}$ National Astronomical Observatory of Japan, 2-21-1 Osawa, Mitaka, Tokyo 181-8588, Japan \\ 13 Astronomical Institute, Osaka Kyoiku University, Asahigaoka, Kashiwara, Osaka 582-8582, Japan
}

A\&A, 523, A17 (2010), https://doi.org/10.1051/0004-6361/200913413

Key words. stars: abundances - galaxies: dwarf - galaxies: evolution - galaxies: formation - galaxies: stellar content errata, addenda

\section{Introduction}

Due to an unfortunate typo in Fortran I/O formats, incorrect values of the collisional broadening coefficient were passed to the spectral synthesis code (CALRAI) in our analysis of Fornax red giants stars. Furthermore, we realized that the lanthanum in tables and figures had escaped the HFS corrections described in Sect. 3.5 of the paper. Here we provide an updated table with all corrected abundances for 72 Fornax stars. The changes are small and non-systematic for most elements, except barium and lanthanum where they are more visible. We thus also reproduce here Figs. 8, 14, 16, and 20 which are the only figures with visible changes. All of our conclusions still hold, although for $\mathrm{Ba}$ and $\mathrm{La}$, the overabundance with respect to the Milky Way are of a smaller magnitude than originally published.

\section{Numerical values in Table A.5}

Key changes from Letarte et al. (2010): the collisional broadening coefficient typo caused the abundance derived from strong lines to be overestimated. Although in principle this concerns all lines, because of our line selection, the net effect on mean abundances is mostly visible for barium abundances, in metalrich stars. The lack of HFS correction on lanthanum lines alters the La abundances slightly, essentially in metal-rich stars. Quantitatively, the average shift (driven by the metal-rich end of our sample) in $[\mathrm{Ba} / \mathrm{Fe}]$ is about $0.2 \mathrm{dex}(\sigma 0.1)$ and $[\mathrm{La} / \mathrm{Fe}]$ is $0.1 \mathrm{dex}$ $(\sigma 0.07)$. All the other elements are affected by an average of less than 0.04 dex. Table A.5 is reproduced here to update the numerical values for all elements in the sample.

\footnotetext{
^ Based on FLAMES observations collected at the European Southern Observatory, proposal number 171.B-0588.
}

The total number of stars that passed all quality tests (see Sect. 3.3 of the original paper) is 72 , as compared to the 81 stars in the original paper. This change is due to slightly inflated uncertainties on some of the diagnostics (slopes with excitation potential or line strength, ionization equilibrium). Tables and figures in this corrigendum therefore only include these 72 Fornax stars.

\section{Updated figures}

Figures 8, 14, 16, and 20 are reproduced here with the updated abundances to highlight the most visible changes in $\mathrm{Ba}$ and La abundances. The effect however changes neither the global trends, nor our interpretation. For barium, our abundances are still higher than those of the Milky Way, but of a smaller amplitude than we first published. For lanthanum too, the overabundance of Fornax with respect to the Milky-Way is reduced.

As illustrated by the revised Fig. 20, the new, lower Ba abundances in Fornax reduce the potential s-process content of $\mathrm{Eu}$ in Fornax, and furthermore no longer allow us to discriminate between the set of different metallicity AGBs yields (Cristallo et al. 2009) for the production of the s-process in Fornax. The final paragraph of Sect. 5.3 should therefore be ignored.

\section{References}

Cristallo, S., Straniero, O., Gallino, R., et al. 2009, ApJ, 696, 797 Johnson, J. A. 2002, ApJS, 139, 219

Letarte, B., Hill, V., Jablonka, P., et al. 2006, A\&A, 453, 547

Letarte, B., Hill, V., Tolstoy, E., et al. 2010, A\&A, 523, A17

Nissen, P. E., \& Schuster, W. J. 1997, A\&A, 326, 751

Simmerer, J., Sneden, C., Cowan, J. J., et al. 2004, ApJ, 617, 1091

Venn, K. A., Irwin, M., Shetrone, M. D., et al. 2004, AJ, 128, 1177 


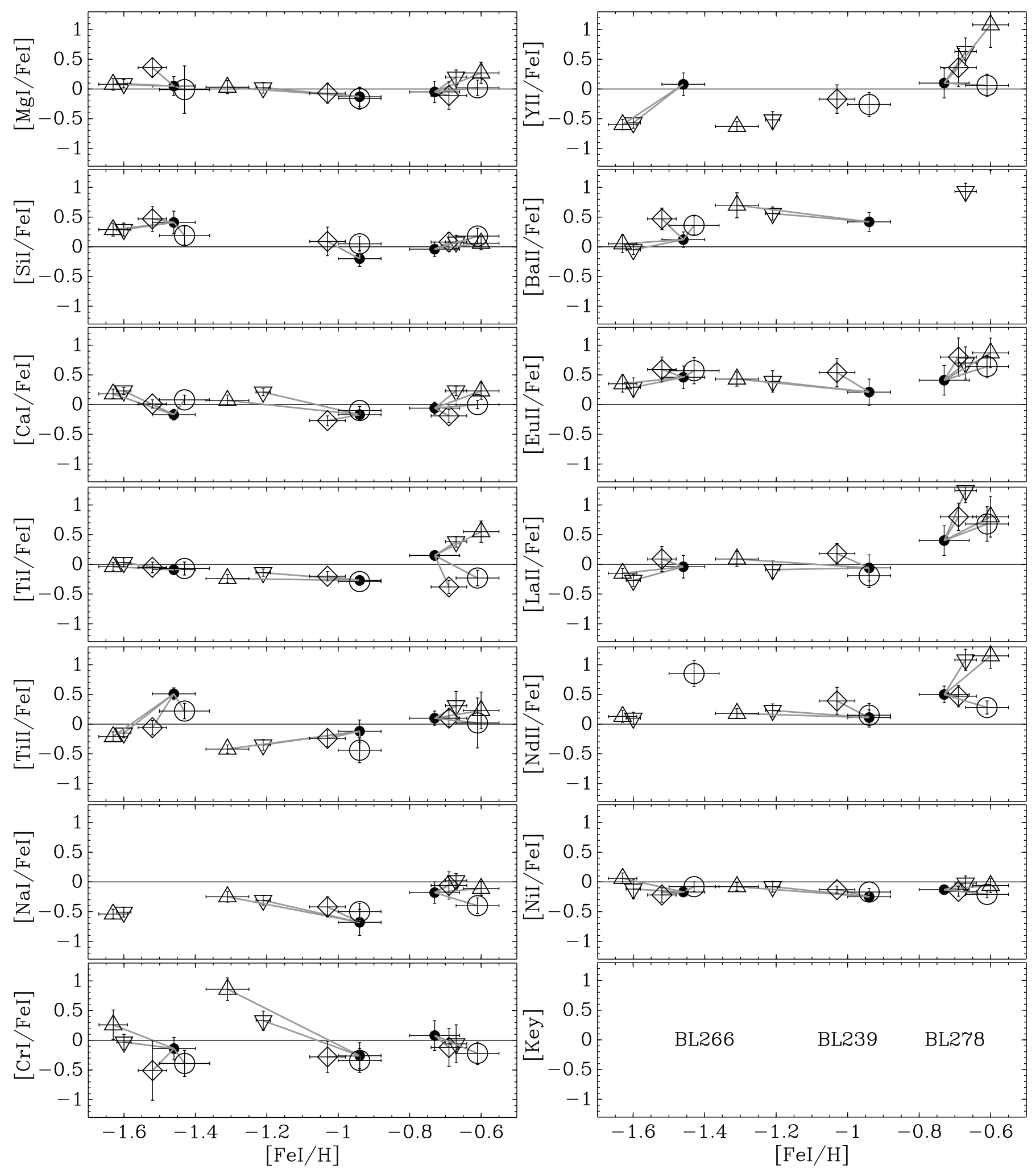

Fig. 8. Abundance ratios for the three stars observed with both UVES (S03) and GIRAFFE (this work) are for the five different checks discussed in Sect. 3.6. These are intented to check the effect of systematics in our analysis. To see which star is which, see the bottom right-hand panel. The GIRAFFE data points are shown as filled circles with lines joining each different check for the same star. The UVES abundances are shown as open symbols. Check 1 results are shown as inverted triangles; Check 2 as triangles; Check 3 as diamonds; Check 4 as circles and Check 5 as small filled dots. 


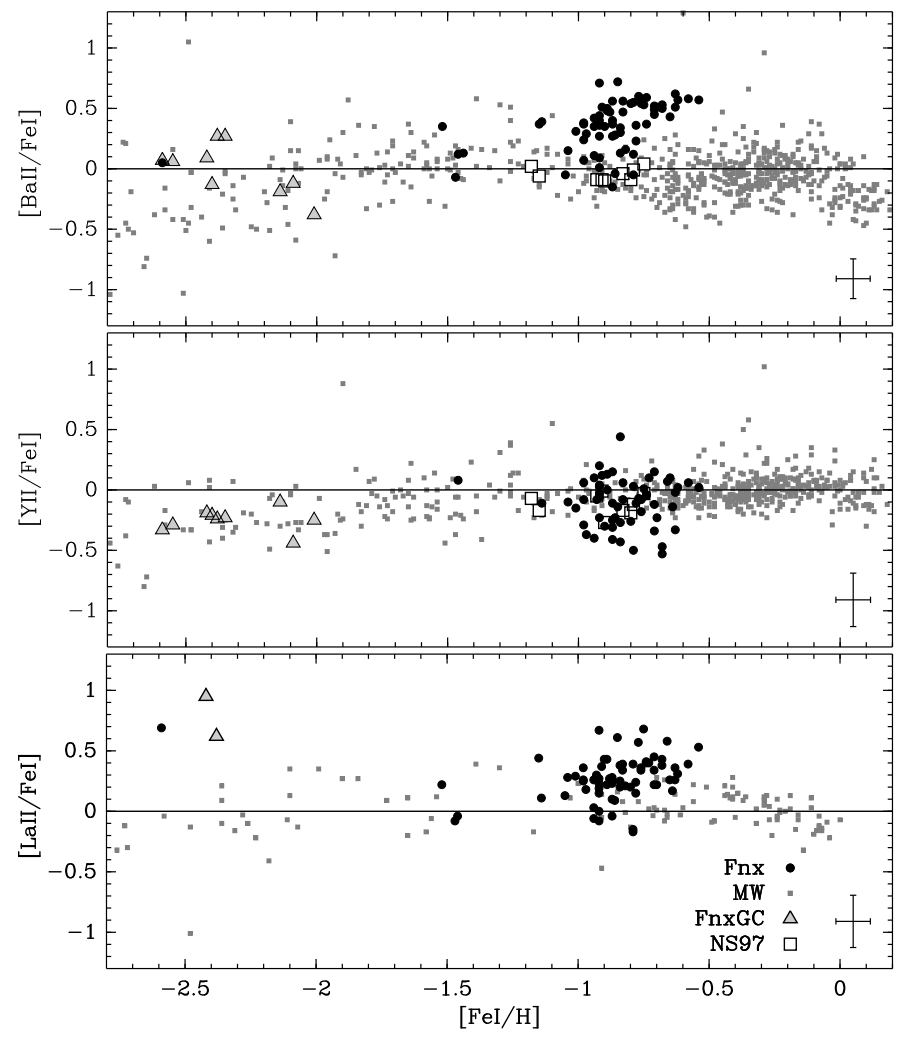

Fig. 14. s-process elements $[\mathrm{Ba} / \mathrm{Fe}],[\mathrm{Y} / \mathrm{Fe}]$, and $[\mathrm{La} / \mathrm{Fe}]$ plotted against $[\mathrm{Fe} / \mathrm{H}]$ for the Fornax field stars. Also plotted for comparison are Galactic stars (original references in Venn et al. 2004) as small grey squares, the Fornax globular clusters (Letarte et al. 2006) as triangles and eight peculiar halo stars (Nissen \& Schuster 1997) as empty squares. In addition we use data from Johnson (2002) and Simmerer et al. (2004) for $[\mathrm{La} / \mathrm{Fe}]$. There is a representative (average) error bar for the Fornax field star abundances in the bottom right corner of each panel. This is the quadratic sum of $[\mathrm{X} / \mathrm{H}]+[\mathrm{Fe} / \mathrm{H}]$, (measurement errors) taken from Table A.5.

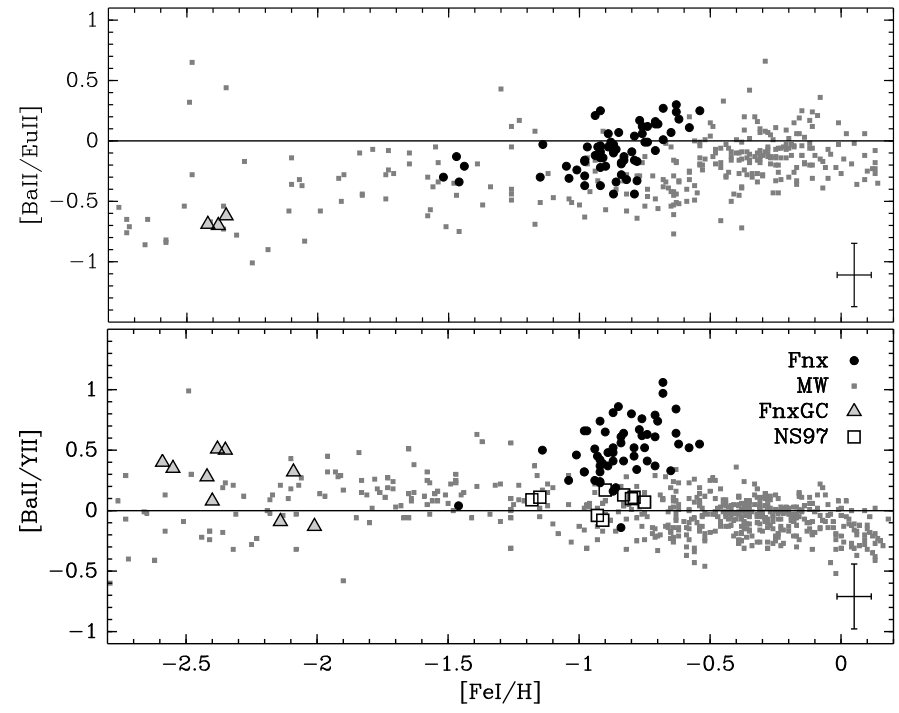

Fig. 16. $[\mathrm{Ba} / \mathrm{Eu}]$ and $[\mathrm{Ba} / \mathrm{Y}]$ as a function of $[\mathrm{Fe} / \mathrm{H}]$ for the Fornax field stars as well as Fornax globulars and MW compilation, as seen in Fig. 14.

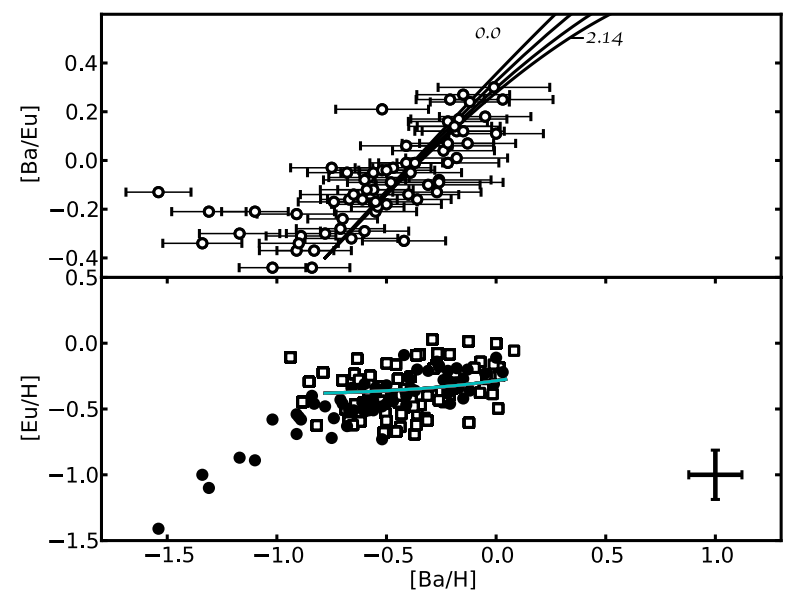

Fig. 20. $[\mathrm{Eu} / \mathrm{H}]$ and $[\mathrm{Ba} / \mathrm{Eu}]$ versus $[\mathrm{Ba} / \mathrm{H}]$. Upper panel: Expected evolution of $[\mathrm{Ba} / \mathrm{Eu}]$ as a function of $[\mathrm{Ba} / \mathrm{H}]$ when the evolution of $\mathrm{Ba}$ and $\mathrm{Eu}$ are triggered by $\mathrm{AGB}$ winds only, starting at $[\mathrm{Ba} / \mathrm{H}]=-0.78$ and $[\mathrm{Ba} / \mathrm{Eu}]=-0.4$. The five solid lines follow the chemical evolution induced by the AGB models of Cristallo et al. (2009) at different metallicities $\left(\left[Z / Z_{\odot}\right]\right)$, which values are indicated close to the upper edges of the model lines. The Fornax sample stars from this work are shown in open circles, together with the individual error bars in $[\mathrm{Ba} / \mathrm{H}]$. Lower panel: Black points show the observations of Fornax stars from this work. The solid line traces the locus of the evolution in $\mathrm{Eu}$ and $\mathrm{Ba}$ induced by the yields of $\left[Z / Z_{\odot}\right]=-2.14$ AGBs (Cristallo et al. 2009), starting at $[\mathrm{Ba} / \mathrm{H}]=-0.78$ and $[\mathrm{Ba} / \mathrm{Eu}]=-0.4$. The black open squares are 85 random trials taking into account the mean observational errors, $0.15 \mathrm{dex}$ in $[\mathrm{Ba} / \mathrm{H}]$ and $0.19 \mathrm{dex}$ in $[\mathrm{Eu} / \mathrm{H}]$ indicated at the bottom right of the frame. They show the scattering of the single model line induced by the observational uncertainties only. 
Table A.5. Abundance ratio of the Fornax Field stars.

\begin{tabular}{|c|c|c|c|c|c|c|c|c|}
\hline $\operatorname{tar}$ & (n) & (n) & n) & $\lceil\mathrm{Ca}$ & $\lceil\mathrm{T} \mathrm{i}$ & $\lceil\mathrm{Ti}$ & $\sigma(n)$ & $\lceil\mathrm{Fe}$ \\
\hline 038 & $-0.43 \pm 0.17(2)$ & $0.04 \pm 0.17$ (2) & $0.35 \pm 0.14$ & $-0.22 \pm 0.10(8)$ & $-0.09 \pm 0.10(6)$ & $0.21 \pm 0.14(3)$ & $-0.09 \pm 0.24(1)$ & $-0.91 \pm 0.07$ \\
\hline & $\ldots \pm \ldots(0)$ & $.06 \pm 0.12(2)$ & $3 \pm 0.16(2)$ & & -0.25 & $-0.03 \pm 0.10(4)$ & $-0.17 \pm 0.16(1)$ & $-1.05 \pm$ \\
\hline 065 & $\ldots \pm \ldots(0)$ & $.02 \pm 0.18$ & $5 \pm 0.16(2)$ & & & & $-0.30 \pm 0.18(1)$ & $-1.44 \pm 0.06$ \\
\hline BL076 & $0.66 \pm 0.13(2)$ & $0.18 \pm 0.13(2)$ & $-0.15 \pm 0.11$ & $-0.20 \pm 0.07(8)$ & $-0.23 \pm 0.06(9)$ & $0.10 \pm 0.15$ & $-0.22 \pm 0.19(1)$ & $-0.86 \pm 0.05$ \\
\hline & & & & & & & & \\
\hline BL081 & $-0.69 \pm 0.13$ & & & & & & $-0.32 \pm 0.19(1)$ & \\
\hline BL084 & $\ldots \pm \ldots(0)$ & $-0.13 \pm 0.18$ & $-0.09 \pm 0.17(2)$ & $-0.25 \pm 0.06$ & $-0.33 \pm 0.06(9)$ & $0.25 \pm 0.16(2)$ & $-0.36 \pm 0.18$ & $-0.87 \pm 0.05$ \\
\hline L085 & $\ldots \pm \ldots(0)$ & $38 \pm 0.15(1)$ & $0.85 \pm 0.15$ & $0.34 \pm 0.06$ & $0.57 \pm 0.15$ & $0.54 \pm 0.22$ & $\ldots \pm \ldots(0)$ & \\
\hline BL091 & $0.45 \pm 0.12(2)$ & $-0.04 \pm 0.12$ & $-0.03 \pm 0.15$ & $-0.08 \pm 0.06(7)$ & $-0.23 \pm 0.06(9)$ & $0.27 \pm 0.17$ & $-0.12 \pm 0.17$ & $-0.98 \pm 0.06$ \\
\hline BL092 & $-0.98 \pm 0.15$ & $0.00 \pm$ & $0.10(4)$ & $-0.22 \pm$ & $-0.24 \pm$ & $0.16(3)$ & $-0.23 \pm 0.21(1)$ & \\
\hline 096 & $-0.82 \pm 0.18$ & $-0.02 \pm 0.14$ & $-0.10 \pm 0.18$ & $-0.18 \pm$ & $-0.20 \pm 0.09$ & & & \\
\hline L097 & $-0.63 \pm 0.12$ & $0.00 \pm 0.17$ & $-0.08 \pm 0.11(3)$ & $-0.21 \pm 0.08$ & $-0.19 \pm 0.05(10)$ & $0.21 \pm 0.10$ & $-0.35 \pm 0.17(1)$ & $-0.92 \pm 0.05$ \\
\hline 100 & $\ldots \pm \ldots(0)$ & & & & & & & \\
\hline BL104 & $-0.77 \pm 0.21$ & $-0.03 \pm 0.15$ & & $-0.17 \pm$ & & & $-0.53 \pm 0.21$ & \\
\hline BL113 & $-0.35 \pm 0.14$ & $-0.09 \pm 0.20(1)$ & & $-0.05 \pm 0.09$ & & & & $-0.76 \pm$ \\
\hline & $\ldots \pm \ldots(0)$ & & & -0.24 & -0.2 & & & \\
\hline & $-0.73 \pm 0.17(1)$ & & & & & & & \\
\hline 125 & $-0.54 \pm$ & -0 . & -0 . & -0 . & & & & \\
\hline & $-0.92=$ & & & & & & & \\
\hline & $\ldots \pm$ & & & & & & & \\
\hline BL140 & $0.85 \pm 0.17$ (1) & -0 & -0 . & -0 . & & & & \\
\hline & -0.8 & & & & & & & \\
\hline & -0.71 & & & & & & & \\
\hline 149 & -0.54 & & & & & & & \\
\hline 150 & -0.6 & & & & & & & \\
\hline & -0.6 & -0 . & & -0 & & & & \\
\hline & -0.62 & & & & & & & \\
\hline & $\ldots \pm$ & & & & & & & \\
\hline & $-0.61 \pm$ & & & & & & & \\
\hline & -0.67 & & & & & & & \\
\hline 163 & $-0.46=$ & -0 . & -0.2 & -0. & & & & \\
\hline 166 & -0.57 & & & -0 & -0 & & & \\
\hline & -0.45 & & & & & & & \\
\hline BL171 & $+\quad(0)$ & $-0.06 \pm 0.10$ & $+\quad(0)$ & & & & & \\
\hline 73 & $-0.50 \pm$ & & & & & & & \\
\hline & -0.4 & & & & & & & \\
\hline & $\ldots \pm$ & & & & & & & \\
\hline & $0.60 \pm$ & & & & & & & \\
\hline & $-0.92 \pm 0.17$ & & & & & & & \\
\hline & -0.7 & & & & & & & \\
\hline & -0.5 & & & & & & & \\
\hline & $-0.46 \pm 0.13(2)$ & -0 . & & & & & & \\
\hline & -0.5 & & & & & & & \\
\hline & -0.6 & & & & & & & \\
\hline & $-0.65 \pm 0.16(2)$ & & & & & & & \\
\hline & -0.6 & & & & & & & \\
\hline & -0.4 & -0 & & & & -0.1 & & -0 . \\
\hline & -0.7 & & & & & & & \\
\hline & -0.9 & & & & & & & \\
\hline & -0.5 & & & & & & -0 & (43) \\
\hline & -0.8 & & & & & & & \\
\hline & -0 & & & & & & & \\
\hline & & & & & & & & \\
\hline & -0.6 & & & & & & & \\
\hline & $-0.55 \pm$ & & & & -0 & 0 & -0 & -1.14 \\
\hline & & & & & & & & \\
\hline & & & & & & & & \\
\hline & $-0.54=$ & $\delta \pm 0.10(3)$ & 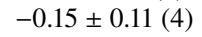 & -0 & -0 & -0 & -0 & -0.70 \\
\hline & & & & & & & & \\
\hline & & & & & & & & \\
\hline & -0.8 & ) & 0 & & -0 & (4) & -0 & $6(39)$ \\
\hline & $-0.79 \pm 0$ & & & & & & & \\
\hline & 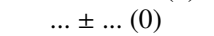 & & & & -( & & & - \\
\hline & $-0.41 \pm 0$ & -0 & -0 & $-c$ & -0 & & (1) & 7 (44) \\
\hline & & & & & & & & \\
\hline & $-c$ & 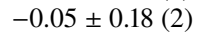 & $2(4)$ & -0 & 0 & 0 & 0. & \\
\hline & & & & -0 & -0 & 1) & & $-1.52 \pm$ \\
\hline & & & & & & & & \\
\hline & $-0.47 \pm 0.12$ & $\pm 0.10(3)$ & $2 \pm 0.10$ & $-0.16 \pm 0.09(7)$ & $-0.04 \pm 0.06(8)$ & $0.09 \pm 0.11$ & $-0.09 \pm 0.17(1)$ & $-0.92 \pm 0.07(39)$ \\
\hline & $-0.72 \pm 0.13$ & $3(2)$ & & & & & & \\
\hline & & & & & & & & \\
\hline BL323 & $-0.72 \pm 0.25$ & $-0.18 \pm 0.14$ & $0.13 \pm 0.18$ & $-0.63 \pm 0.13$ & $-0.31 \pm 0.09(8)$ & $0.04 \pm 0.14$ & $-0.28 \pm 0.25$ & $-0.92 \pm 0.06$ \\
\hline
\end{tabular}

Notes. We list the abundance, its associated error $(\sigma)$ and the number of lines used $(n)$. The quoted error is the error on [element/H], not $[\mathrm{X} / \mathrm{Fe}]$. 
Table A.5. continued.

\begin{tabular}{|c|c|c|c|c|c|c|c|}
\hline $\operatorname{ar}$ & $\underline{\mathrm{H} \mid \mathrm{H}}$ & & & $D^{1 \mathrm{Da}}$ & & & $\sigma(n)$ \\
\hline BL038 & $-0.71 \pm 0.08$ & $-0.12 \pm 0.06(15)$ & $0.12 \pm 0.24(1)$ & $0.51 \pm 0.17(2)$ & $0.53 \pm 0.24(1)$ & $0.25 \pm 0.17$ & $0.91 \pm 0.24(1)$ \\
\hline BL045 & $-0.76 \pm 0.08(5)$ & $-0.21 \pm 0.07(11)$ & $\ldots \pm \ldots(0)$ & $-0.05 \pm 0.14$ & $0.16 \pm 0.16(1)$ & $-0.09 \pm 0.16(1)$ & $0.20 \pm 0.16(1)$ \\
\hline BL065 & $-1.06 \pm 0.15(5)$ & $-0.15 \pm 0.07(8)$ & $\ldots \pm \ldots(0)$ & $0.13 \pm 0.13$ & $\ldots \pm \ldots(0)$ & $0.50 \pm 0.18$ & $0.37 \pm 0.18$ \\
\hline BL076 & $-0.43 \pm 0.12$ & $-0.25 \pm 0.08$ & $\ldots \pm \ldots(0)$ & $0.28 \pm 0.13(2)$ & $\ldots \pm \ldots(0)$ & $0.21 \pm 0.12$ & $0.46 \pm 0.19(1)$ \\
\hline BL077 & $-0.40 \pm 0.21$ & $-0.24 \pm 0.06$ & $\ldots \pm \ldots(0)$ & $0.12 \pm 0.10(2)$ & $-0.12 \pm 0.14$ & $0.23 \pm 0.11$ & $0.39 \pm 0.14(1)$ \\
\hline BL081 & $-0.44 \pm 0.17$ & $-0.24 \pm 0.06(14)$ & $-0.33 \pm 0.19(1)$ & $0.51 \pm 0.13(2)$ & $0.41 \pm 0.19$ & $0.26 \pm 0.14(3)$ & $0.41 \pm 0.19$ \\
\hline BL084 & $-0.67 \pm 0.09$ & $-0.21 \pm 0.06(13)$ & $-0.41 \pm 0.18(1)$ & $0.40 \pm 0.13(2)$ & $0.19 \pm 0.18$ & $0.21 \pm 0.14$ & $0.59 \pm 0.18$ \\
\hline BL085 & $-2.01 \pm 0.10$ & $0.72 \pm 0.22(5)$ & $\ldots \pm \ldots(0)$ & $0.05 \pm 0.11(2)$ & $0.71 \pm 0.15$ & $1.49 \pm 0.15$ & $\ldots \pm \ldots(0)$ \\
\hline BL091 & $-0.62 \pm 0.19(5)$ & $-0.09 \pm 0.07(13)$ & $-0.29 \pm 0.17$ & $0.37 \pm 0.12(2)$ & $0.31 \pm 0.17(1)$ & $0.45 \pm 0.10$ & $0.66 \pm 0.17$ \\
\hline BL092 & $-0.54 \pm 0.09(3)$ & $-0.21 \pm 0.09$ & $0.20 \pm 0.21$ & $0.44 \pm 0.15(2)$ & $0.27 \pm 0.21$ & $0.28 \pm 0.12$ & $0.68 \pm 0.21(1)$ \\
\hline BL096 & $-0.91 \pm 0.18$ & $-0.31 \pm 0.08(12)$ & $-0.34 \pm 0.25$ & $0.45 \pm 0.18(2)$ & $0.35 \pm 0.25$ & $0.29 \pm 0.18$ & $0.76 \pm 0.25(1)$ \\
\hline BL097 & $-0.54 \pm 0.21(5)$ & $-0.08 \pm 0.06(16)$ & $-0.06 \pm 0.17$ & $0.36 \pm 0.12(2)$ & $0.21 \pm 0.17(1)$ & $0.30 \pm 0.10$ & $0.62 \pm 0.17(1)$ \\
\hline BL100 & $-0.68 \pm 0.12(5)$ & $-0.26 \pm 0.07(15)$ & $-0.40 \pm 0.19(1)$ & $0.11 \pm 0.16(2)$ & $0.07 \pm 0.19$ & $0.31 \pm 0.13$ & $\ldots \pm \ldots(0)$ \\
\hline BL104 & $-0.34 \pm 0.20$ & $-0.14 \pm 0.07(12)$ & $0.06 \pm 0.21(1)$ & $0.38 \pm 0.15(2)$ & $0.47 \pm 0.21(1)$ & $0.36 \pm 0.12(3)$ & $0.88 \pm 0.21(1)$ \\
\hline BL113 & $-0.65 \pm 0.10(5)$ & $-0.23 \pm 0.06(12)$ & $-0.18 \pm 0.20(1)$ & $0.58 \pm 0.20(1)$ & $0.51 \pm 0.20$ & $0.25 \pm 0.15$ & $0.65 \pm 0.20$ \\
\hline BL115 & $-0.95 \pm 0.10$ & $-0.16 \pm 0.07(10)$ & $\ldots \pm \ldots(0)$ & $-0.07 \pm 0.11$ & $-0.06 \pm 0.16(1)$ & $0.43 \pm 0.16(1)$ & $0.07 \pm 0.16(1)$ \\
\hline BL123 & $-0.82 \pm 0.07$ & $-0.16 \pm 0.05(16)$ & $-0.08 \pm 0.17(1)$ & $0.24 \pm 0.12(2)$ & $0.37 \pm 0.17(1)$ & $0.32 \pm 0.10$ & $0.53 \pm 0.17(1)$ \\
\hline BL125 & $-0.61 \pm 0.10$ & $-0.31 \pm 0.06(15)$ & $0.01 \pm 0.24$ & $0.53 \pm 0.17(2)$ & $0.93 \pm 0.24(1)$ & $0.40 \pm 0.14$ & $0.76 \pm 0.24(1)$ \\
\hline BL132 & $-0.70 \pm 0.10$ & $-0.20 \pm 0.07(12)$ & $\ldots \pm \ldots(0)$ & $0.01 \pm 0.13(2)$ & $-0.02 \pm 0.19(1)$ & $0.14 \pm 0.13$ & $0.33 \pm 0.19(1)$ \\
\hline BL138 & $-0.58 \pm 0.13(5)$ & $-0.10 \pm 0.08(14)$ & $-0.15 \pm 0.17(1)$ & $0.31 \pm 0.12(2)$ & $0.42 \pm 0.17$ & $0.23 \pm 0.14$ & $0.73 \pm 0.17(1)$ \\
\hline BL140 & $0.19 \pm 0.24$ & $-0.15 \pm 0.08$ & $0.06 \pm 0.17$ & $0.47 \pm 0.12(2)$ & $0.47 \pm 0.17$ & $0.31 \pm 0.21$ & $0.86 \pm 0.17$ \\
\hline BL141 & $-0.43 \pm 0.10(5)$ & $-0.24 \pm 0.06(15)$ & $-0.43 \pm 0.21(1)$ & $0.13 \pm 0.15$ & $0.28 \pm 0.21(1)$ & $0.27 \pm 0.12$ & $0.54 \pm 0.21(1)$ \\
\hline BL146 & $-0.65 \pm 0.07(5)$ & $-0.18 \pm 0.06(13)$ & $-0.08 \pm 0.16(1)$ & $0.37 \pm 0.11$ & $0.40 \pm 0.16(1)$ & $0.28 \pm 0.10$ & $0.53 \pm 0.16(1)$ \\
\hline BL149 & $-0.81 \pm 0.15(5)$ & $-0.26 \pm 0.08(13)$ & $0.03 \pm 0.25$ & $0.40 \pm 0.18(2)$ & $0.30 \pm 0.25(1)$ & $0.06 \pm 0.25$ & $0.55 \pm 0.25$ \\
\hline BL150 & $-0.41 \pm 0.12$ & $-0.30 \pm 0.08$ & $\ldots \pm \ldots(0)$ & $0.16 \pm 0.16(2)$ & $0.31 \pm 0.22$ & $0.40 \pm 0.16$ & $0.66 \pm 0.22(1)$ \\
\hline BL151 & $-0.61 \pm 0.09$ & $-0.18 \pm 0.05$ & $\ldots \pm \ldots(0)$ & $0.47 \pm 0.13(2)$ & $0.37 \pm 0.18$ & $0.33 \pm 0.10$ & $0.62 \pm 0.18(1)$ \\
\hline BL155 & $-0.49 \pm 0.17$ & $-0.12 \pm 0.09(15)$ & $0.15 \pm 0.22$ & $0.52 \pm 0.16(2)$ & $0.64 \pm 0.22(1)$ & $0.63 \pm 0.26(3)$ & $0.53 \pm 0.22(1)$ \\
\hline BL156 & $-0.63 \pm 0.17(5)$ & $-0.07 \pm 0.08$ & $\ldots \pm \ldots(0)$ & $0.37 \pm 0.13(2)$ & $0.53 \pm 0.19$ & $0.42 \pm 0.23(2)$ & $0.81 \pm 0.19(1)$ \\
\hline BL158 & $-0.35 \pm 0.15$ & $-0.17 \pm 0.08$ & $0.14 \pm 0.23$ & $0.72 \pm 0.23(1)$ & $0.78 \pm 0.23(1)$ & $0.49 \pm 0.23(3)$ & $0.85 \pm 0.23(1)$ \\
\hline BL160 & $-0.60 \pm 0.07(5)$ & $-0.25 \pm 0.06(16)$ & $-0.11 \pm 0.14$ & $0.37 \pm 0.10(2)$ & $0.40 \pm 0.14$ & $0.38 \pm 0.09(3)$ & $0.55 \pm 0.14$ \\
\hline BL163 & $-0.59 \pm 0.12$ & $-0.16 \pm 0.06(12)$ & $-0.04 \pm 0.20(1)$ & $0.59 \pm 0.20(1)$ & $0.59 \pm 0.20(1)$ & $0.45 \pm 0.12$ & $0.70 \pm 0.20(1)$ \\
\hline BL166 & $-0.54 \pm 0.20$ & $-0.18 \pm 0.07(13)$ & $0.00 \pm 0.22$ & $0.48 \pm 0.16(2)$ & $0.58 \pm 0.22$ & \pm 0.13 & $0.55 \pm 0.22(1)$ \\
\hline BL168 & $-0.57 \pm 0.17$ & $-0.16 \pm 0.06(13)$ & $-0.30 \pm 0.20$ & $0.35 \pm 0.14(2)$ & $0.56 \pm 0.20(1)$ & $0.30 \pm 0.12$ & $0.73 \pm 0.20(1)$ \\
\hline BL171 & $-0.19 \pm 0.12$ & $-0.17 \pm 0.07(14)$ & $-0.23 \pm 0.18$ & $0.09 \pm 0.13(2)$ & $0.36 \pm 0.18$ & $0.30 \pm 0.17$ & $0.59 \pm 0.18$ \\
\hline BL173 & $-0.75 \pm 0.13$ & $-0.25 \pm 0.08(12)$ & $-0.25 \pm 0.20$ & $0.27 \pm 0.14$ & $0.42 \pm 0$ & $0.42 \pm 0.21$ & $20(1)$ \\
\hline BL185 & $-0.78 \pm 0.12$ & $-0.19 \pm 0.08(16)$ & $-0.07 \pm 0.23$ & $0.60 \pm 0.16(2)$ & $0.79 \pm 0.23(1)$ & $0.46 \pm 0.16(2)$ & $0.61 \pm 0.23(1)$ \\
\hline BL190 & $-0.60 \pm 0.10$ & $-0.31 \pm 0.07$ & $-0.50 \pm 0.23(1)$ & $-0.05 \pm 0.13$ & $-0.14 \pm 0.18(1)$ & $0.27 \pm 0.22(2)$ & $0.54 \pm 0.18$ \\
\hline BL195 & $-0.73 \pm 0.08$ & $-0.21 \pm 0.06(8)$ & $\ldots \pm \ldots(0)$ & $0.07 \pm 0.16(2)$ & $\ldots \pm \ldots(0)$ & $0.04 \pm 0.11$ & $0.54 \pm 0.18$ \\
\hline BL196 & $-0.85 \pm 0.09(5)$ & $-0.28 \pm 0.06(11)$ & $-0.10 \pm 0.17(1)$ & $=0.12(2)$ & $0.39 \pm 0.17(1)$ & $=0.10(3)$ & $0.60 \pm 0.17(1)$ \\
\hline BL197 & $-0.79 \pm 0.13$ & $-0.13 \pm 0.07(13)$ & $0.13 \pm 0.24$ & $0.17(2)$ & $0.34 \pm$ & $0.24 \pm 0.17$ (2) & $0.76 \pm 0.24(1)$ \\
\hline BL203 & $-0.55 \pm 0.10$ & $-0.21 \pm 0.08$ & $-0.27 \pm 0.26(1)$ & $0.34 \pm 0.18$ & $0.35 \pm 0.26$ & $0.31 \pm 0.19$ & $0.70 \pm 0.26$ \\
\hline BL205 & $-0.65 \pm 0.07$ & $-0.22 \pm 0.06$ & $-0.12 \pm 0.19$ & $0.49 \pm 0.13(2)$ & $0.46 \pm 0.19$ & $=0.21(2)$ & $0.46 \pm 0.19$ \\
\hline BL208 & $-0.74 \pm 0.06(3)$ & $-0.29 \pm 0.06(16)$ & $-0.53 \pm 0.22$ & $0.16(2)$ & $0.60 \pm 0$ & $0.38 \pm 0.13$ & $0.37 \pm 0.22$ \\
\hline BL210 & $-0.35 \pm 0.20$ & $-0.12 \pm 0.07(13)$ & $-0.08 \pm 0.22(1)$ & $0.54 \pm 0.16(2)$ & $0.50 \pm 0.22$ & $0.50 \pm 0.13$ & $0.67 \pm 0.22(1)$ \\
\hline BL211 & $-0.92 \pm 0.15$ & $-0.19 \pm 0.07(16)$ & $0.02 \pm 0.22(1)$ & $0.57 \pm 0.16(2)$ & $0.49 \pm 0.22$ & $0.28 \pm 0.13$ & $0.59 \pm 0.22$ \\
\hline BL213 & $-0.78 \pm 0.10(5)$ & $-0.27 \pm 0.06(15)$ & $0.10 \pm 0.22(1)$ & $0.35 \pm 0.16(2)$ & $0.39 \pm 0.22$ & $0.31 \pm 0.16(2)$ & $0.65 \pm 0.22(1)$ \\
\hline BL216 & $-1.06 \pm 0.17(2)$ & $-0.11 \pm 0.07(14)$ & $0.03 \pm 0.24(1)$ & $5 \pm 0.17(2)$ & $0.60 \pm 0.24(1)$ & $1 \pm 0.14(3)$ & $0.77=$ \\
\hline BL218 & $-0.59 \pm 0.18$ & $-0.16 \pm 0.08(16)$ & $-0.02 \pm 0.27(1)$ & $0.62 \pm 0.27(1)$ & $0.57 \pm 0.27(1)$ & $0.16(3)$ & $0.50 \pm 0.27(1)$ \\
\hline BL221 & $-0.74 \pm 0.18$ & $-0.26 \pm 0.08(15)$ & $-0.08 \pm 0.21$ & $0.56 \pm 0.15(2)$ & $0.52 \pm 0.21$ & $0.48 \pm 0.16(2)$ & $0.94 \pm 0.21$ \\
\hline BL227 & $-0.65 \pm 0.14$ (3) & $-0.23 \pm 0.07(12)$ & $0.15 \pm 0.25$ & $0.56 \pm 0.18$ & $0.31 \pm$ & $0.39 \pm 0.25$ & $0.87 \pm 0.25$ \\
\hline BL228 & $-0.44 \pm 0.14$ & $-0.26 \pm 0.07$ & $-0.31 \pm 0.17(1)$ & $-0.15 \pm 0.11(2)$ & $0.01 \pm 0$ & $0.41 \pm 0.16$ & $0.40 \pm$ \\
\hline BL229 & $-0.44 \pm 0.13$ & $-0.27 \pm 0.09$ & $-0.47 \pm 0.24(1)$ & $0.50 \pm 0.17$ (2) & $0.57 \pm 0.24(1)$ & $0.39 \pm 0.14$ & $0.72 \pm 0.24$ \\
\hline BL233 & $-0.43 \pm 0.10$ & $-0.24 \pm 0.08(15)$ & $0.10 \pm 0.22$ & $0.43 \pm 0.16(2)$ & $0.41 \pm 0.22$ & $0.24 \pm 0.13$ & $0.52 \pm 0.22(1)$ \\
\hline BL239 & $-0.79 \pm 0.10$ & $-0.25 \pm 0.08$ & $\ldots \pm \ldots(0)$ & $0.42 \pm 0.16(2)$ & $-0.03 \pm 0.22(1)$ & $0.11 \pm 0.13(3)$ & $0.28 \pm 0.22(1)$ \\
\hline BL242 & $-0.93 \pm 0.18$ & $-0.13 \pm 0.06(13)$ & $-0.11 \pm 0.20$ & $0.39 \pm 0.14(2)$ & $0.15 \pm 0.20(1)$ & $0.29 \pm 0.23(2)$ & $0.51 \pm 0.20(1)$ \\
\hline BL247 & $-0.60 \pm 0.12(5)$ & $-0.05 \pm 0.08(15)$ & $0.44 \pm 0.15$ & $0.30 \pm 0.11(2)$ & $0.53 \pm 0.15$ & $0.95 \pm 0.11(2)$ & $0.66 \pm 0.15$ \\
\hline BL250 & $-0.46 \pm 0.30$ & $-0.15 \pm 0.10$ & $-0.14 \pm 0.26(1)$ & $\ldots \pm \ldots(0)$ & $6(1)$ & $0.18(3)$ & $26(1)$ \\
\hline BL253 & $-0.74 \pm 0.10$ & $-0.22 \pm 0.07$ & $-0.23 \pm 0.18$ & $0.51 \pm 0.13(2)$ & $0.39 \pm 0.18$ & $0.32 \pm 0.17$ & $0.58 \pm 0.18$ \\
\hline BL257 & $-1.07 \pm 0.12(3)$ & $-0.21 \pm 0.07$ & $0.02 \pm 0.24$ & $0.57 \pm 0.24(1)$ & $0.80 \pm 0.24(1)$ & $0.29 \pm 0.14$ & $0.52 \pm 0.24$ \\
\hline BL258 & $-0.62 \pm 0.08$ & $-0.20 \pm 0.07(15)$ & $0.06 \pm 0.23$ & $0.58 \pm 0.23(1)$ & $0.61 \pm 0.23$ & $0.45 \pm 0.28$ & $0.75 \pm 0.23$ \\
\hline BL260 & $-0.39 \pm 0.11$ & $-0.29 \pm 0.07(12)$ & $-0.23 \pm 0.19(1)$ & $-0.04 \pm 0.13(2)$ & $0.17 \pm 0.19$ & $0.34 \pm 0.12$ & $0.41 \pm 0.19$ \\
\hline BL261 & $-0.45 \pm 0.22$ & $-0.31 \pm 0.10(12)$ & $-0.11 \pm 0.21$ & $0.23 \pm 0.15(2)$ & $0.25 \pm 0.21$ & $0.35 \pm 0.12$ & $0.55 \pm 0.21(1)$ \\
\hline BL266 & $-1.17 \pm 0.18$ & $-0.17 \pm 0.07(10)$ & $0.08 \pm 0.19$ & $0.12 \pm 0.13(2)$ & $-0.02 \pm 0.19$ & $\ldots \pm \ldots(0)$ & $0.50 \pm 0.19$ \\
\hline BL267 & $-0.65 \pm 0.11(5)$ & $-0.18 \pm 0.09$ & $-0.04 \pm 0.18$ & $0.37 \pm 0.13(2)$ & $0.57 \pm 0.18$ & $0.09 \pm 0.18$ & $0.54 \pm 0.18$ \\
\hline BL269 & $-0.48 \pm 0.28$ & $-0.24 \pm 0.10(15)$ & $-0.26 \pm 0.30(1)$ & $0.54 \pm 0.21(2)$ & $0.30 \pm 0.30(1)$ & $0.26 \pm 0.17$ & $0.87 \pm 0.30(1)$ \\
\hline BL278 & $-0.86 \pm 0.13$ & $-0.13 \pm 0.07$ & $0.10 \pm 0.25(1)$ & $\ldots \pm \ldots(0)$ & $0.62 \pm 0.25(1)$ & $0.50 \pm 0.14$ & $0.63 \pm 0.25$ \\
\hline BL279 & $-1.31 \pm 0.16(3)$ & $-0.02 \pm 0.10(12)$ & & $0.35 \pm 0.14$ (2) & $0.24 \pm 0.20$ & $0.67 \pm 0.20(1)$ & $0.70 \pm 0.20(1)$ \\
\hline BL295 & $-0.23 \pm 0.22(3)$ & $-0.19 \pm 0.07(15)$ & $0.07 \pm 0.26(1)$ & . (0) & $0.86 \pm 0.26(1)$ & $0.29 \pm 0.18$ & $0.68 \pm 0.26(1)$ \\
\hline BL300 & $-0.58 \pm 0.09$ & $-0.16 \pm 0.07(14)$ & $-0.03 \pm 0.17(1)$ & $0.71 \pm 0.12(2)$ & $0.91 \pm 0.17(1)$ & $0.49 \pm 0.10$ & $0.61 \pm 0.17(1)$ \\
\hline BL304 & $-0.84 \pm 0.10$ & $-0.19 \pm 0.07(14)$ & $-0.37 \pm 0.19(1)$ & $0.29 \pm 0.13(2)$ & $0.31 \pm 0.19$ & $0.18 \pm 0.11$ & $0.48 \pm 0.19$ \\
\hline BL315 & $-0.56 \pm 0.16(4)$ & $-0.39 \pm 0.11(15)$ & $\ldots \pm \ldots(0)$ & $0.36 \pm 0.15(2)$ & $0.32 \pm 0.21(1)$ & $0.32 \pm 0.21(1)$ & $0.90 \pm 0.21(1)$ \\
\hline BL323 & $-0.79 \pm 0.16(3)$ & $-0.22 \pm 0.07(15)$ & $0.04 \pm 0.25$ & $0.27 \pm 0.18(2)$ & $0.09 \pm 0.25(1)$ & $0.14 \pm 0.19$ & $0.59 \pm 0.25$ \\
\hline
\end{tabular}

